\title{
Corrections to: The Effect of Nordic Hamstring Exercise Intervention Volume on Eccentric Strength and Muscle Architecture Adaptations: A Systematic Review and Meta-analyses
}

\author{
Matthew Cuthbert ${ }^{1,2}$ - Nicholas Ripley ${ }^{1} \cdot$ John J. McMahon ${ }^{1} \cdot$ Martin Evans $^{2} \cdot$ G. Gregory Haff $^{1,3} \cdot$ Paul Comfort $^{1,4}$
}

Published online: 7 November 2019

(c) The Author(s) 2019

Correction to: Sports Medicine https://doi.org/10.1007/s40279-019-01178-7

Page 5, column 1, section 3.2, paragraph 1, sentence 1: The following sentence, which previously read:

"Consistency between the studies assessed for both hamstring strength measures and muscle architecture was moderate to high, with $I^{2}$ values of $62.49 \%$ and $88.03 \%$, respectively."

Should read:

"Consistency between the studies assessed for both hamstring strength measures and muscle architecture was moderate to high, with $I^{2}$ values of $58.58 \%$ and $88.03 \%$, respectively."

Page 5, columns 1-2, section 3.2, paragraph 1, sentence 3: The following sentence, which previously read:

"Two risk of bias assessments were also performed, the first (Cochrane risk of bias assessment tool) showing a low

The original article can be found online at https://doi.org/10.1007/ s40279-019-01178-7.

Matthew Cuthbert

m.cuthbert@edu.salford.ac.uk

1 Human Performance Laboratory, University of Salford, Greater Manchester, UK

2 The FA Group, St George's Park, Burton-upon-Trent, Staffordshire, UK

3 Centre for Exercise and Sports Sciences Research (CESSR), School of Exercise and Health Sciences, Edith Cowan University, Joondalup, Australia

4 Institute for Sport, Physical Activity and Leisure, Carnegie School of Sport, Leeds Beckett University, Leeds, UK risk of bias overall within the randomized controlled studies included in this review (Fig. 2), the second identifying the results of this meta-analysis are not subject to publication bias $(p<0.001)$ with 250 and 663 "filed-away" studies needed to prove null effects of NHE interventions on strength and architecture, respectively."

Should read:

"Two risk of bias assessments were also performed, the first (Cochrane risk of bias assessment tool) showing a low risk of bias overall within the randomized controlled studies included in this review (Fig. 2), the second identifying the results of this meta-analysis are not subject to publication bias $(p<0.001)$ with 178 and 663 "filed-away" studies needed to prove null effects of NHE interventions on strength and architecture, respectively."

Pages 5-6, columns 2 (page 5) and 1 (page 6), section 3.3, paragraph 1, sentence 6: The following sentence, which previously read:

"The pooled summary of variance from the random-effects model was 0.374 ( $p=0.009,95 \%$ CI $0.94-0.655)$ for strength and 0.793 ( $p<0.001,95 \%$ CI $0.338-1.248)$ for muscle architecture.”

Should read:

"The pooled summary of variance from the random-effects model was $0.439(p=0.001,95 \%$ CI $0.160-0.709)$ for strength and 0.793 ( $p<0.001,95 \%$ CI $0.338-1.248)$ for muscle architecture."

Page 11, Table 1, Alt et al. [58] row: The cell entry in column 1 , which previously read: 
"Alt et al. [58]"

Should read:

"Alt et al. [48]"
Open Access This article is distributed under the terms of the Creative Commons Attribution 4.0 International License (http://creativeco mmons.org/licenses/by/4.0/), which permits unrestricted use, distribution, and reproduction in any medium, provided you give appropriate credit to the original author(s) and the source, provide a link to the Creative Commons license, and indicate if changes were made. 